\title{
Quantization as a guide to ontic structure
}

\author{
Karim Thébault* \\ Munich Center for Mathematical Philosophy, \\ Ludwig Maximilians Universität, Ludwigstrasse 31, D-80539, Munich, Germany
}

March 23, 2014

\begin{abstract}
The ontic structural realist stance is motivated by a desire to do philosophical justice to the success of science, whilst withstanding the metaphysical undermining generated by the various species of ontological underdetermination. We are, however, as yet in want of general principles to provide a scaffold for the explicit construction of structural ontologies. Here we will attempt to bridge this gap by utilising the formal procedure of quantization as a guide to ontic structure of modern physical theory. The example of non-relativistic particle mechanics will be considered and, for that case, it will be shown that a viable candidate for an OSR framework can be constituted in terms of the combination of a state space, with Poisson bracket structure, and a set of observables, with Lie algebra structure.
\end{abstract}

Keywords: Structural Realism; Ontology; Quantization; Underdetermination

\section{Contents}

\begin{tabular}{lll}
\hline 1 & Introduction & 2
\end{tabular}

2 Formulation Underdetermination and Structural Ontologies 3

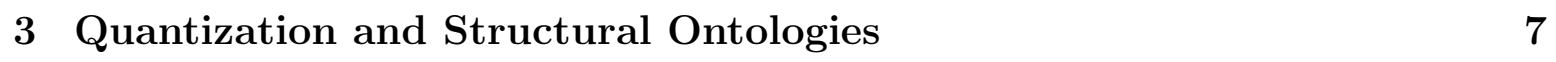

4 The Case of Non-Relativistic Particle Mechanics 9

4.1 Formulation Underdetermination . . . . . . . . . . . . . . . . . . . . . . . . . . . 9

4.2 The Classical Ontology . . . . . . . . . . . . . . . . . . . . . . . . . . . 12

4.3 Quantum Theory and the Generalized Structural Ontology . . . . . . . 13

4.4 Interpretation of Results . . . . . . . . . . . . . . 16

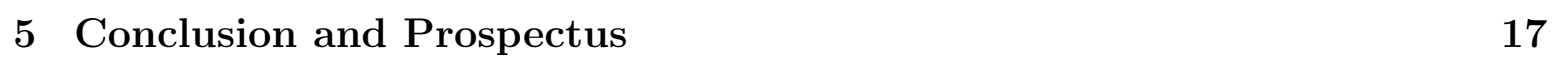

*email: karim.thebault@gmail.com 


\section{Introduction}

Ontic structural realism (OSR) (Ladyman 1998; French and Ladyman 2003; Ladyman and Ross 2007) is in part motivated by two arguments towards the underdetermination of the ontology associated with traditional realist descriptions of science 1 The first is formulation underdetermination, and springs from the multiplicity of empirically equivalent formulations of a given theory (Jones 1991; Bain 2009; French 2011). The second is pessimistic meta-induction, and springs from the historical superseding of one empirically well-confirmed theory by another (Laudan 1981; Worrall 1989; Ladyman 1998). Under OSR the ontology of a physical theory is constituted by mathematical structures rather than objects and entities. To avoid formulation underdetermination, one is limited to an ontology constituted by the structures common between two different formulations of a theory; and to avoid pessimistic meta-induction one is limited to the structures common to a theory and its successor. For OSR to be viable: i) these structures must be substantial enough to constitute a viable alternative ontology; and ii) the structures used to avoid the two arguments must be consistent.

Here we will construct a proposal to evaluate OSR with respect to i) and ii) in the context of the mathematical structures which underlie quantization (the procedure for turning a classical theory into a quantum theory). It will be argued that through the close investigation of the formal techniques that lie behind quantization, we can gain an insight into certain fundamental construction principles which serve to guide us in the difficult task of building a substantive and consistent ontic structural framework for physical theory. The first step in the implementation of this proposal is the consideration of the case of non-relativistic particle mechanics. We will investigate the Lagrangian and Hamiltonian formulations of non-relativistic particle mechanics, together with the powerful and general technique of geometric quantization. The idea is to determine whether we can isolate the structures that both connect and generalize the relationships between the various classical and quantum formal structures. We will show that a viable candidate for an OSR framework can be constituted in terms of the combination of a state space, with Poisson bracket structure, and a set of observables, with Lie algebra structure. Future work will consider gauge theories, in particular classical/quantum field theories, with a view towards exploring the problem of quantizing gravity within our particular perspective on structuralism.

Our purpose here will not be to defend the necessity of the various arguments towards underdetermination of ontology or the ontic structural realist response. And we will also here neglect consideration of the epistemic variant of structural realism (Worrall 1989) (although we note that much of the structure identified in the analysis below would seem to be reconcilable with either position). Rather our present analysis will examine, in as concrete and explicit terms as possible, whether a viable candidate for structural ontology can or cannot be constructed in the context of an explicit case study. If it transpires that the proponent of OSR is not able to point to a consistent, generalising and dynamical structural framework, in even the simplest of cases, then the position will be substantially undermined, irrespective of the strength or weakness of the relevant motivating arguments. Conversely, if a suitable framework can be found, then we have good grounds to examine that framework's extension to more general theories,

\footnotetext{
${ }^{1}$ These are also taken as motivations for the 'epistemic' variant of structural realism which will not be discussed here. See (Frigg and Votsis 2011) for an excellent overview of both positions.
} 
over and above the question of granting it a privileged metaphysical status. In the end, if the pursuit of the principles underlying the consistent construction of structural ontology gives us any genuine insight into the foundations of physical theory, then it will have proved a worthwhile endeavour.

\section{Formulation Underdetermination and Structural Ontologies}

Following the analysis of French (2011), we can consider three scenarios which can be grouped together under the heading ontological (or metaphysical) underdetermination: theoretical underdetermination, interpretational underdetermination and formulation underdetermination. The first is the most familiar within the philosophy of science and is when we are presented with distinct theories each consistent with the same set of phenomena but each entailing an ontology incompatible with the other. A classic example of such an underdetermination case is that between special relativity and Lorentzian ether theory. ${ }^{2}$

The second interpretational notion of underdetermination is particularly familiar within the philosophy of physics and relates to the existence or two or more competing candidate ontologies associated with the same physico-mathematical formalism. An interpretation of a theory is an assignment of representational capacity to certain aspects of the formalism (with the scope of this assignment and nature of this capacity depending upon one's framework for understanding scientific theory and representation in general). Interpretational underdetermination is then the scenario whereby there are multiple distinct representational assignments consistent with the same formal and empirical structures - i.e., 'the representational relationship between formalism and worlds in one-to-many' (Rickles 2008, p.8). A classic example of such underdetermination is in the debate regarding the spacetime ontology of classical theories of physics. In theories such as Newtonian mechanics or general relativity it is found that one is able to interpret the same formalism as representing universes either with or without spacetime points, and thus the spacetime ontology of these theories is radically underdetermined by interpretational choice.

The third variant of underdetermination is perhaps the most neglected and shall be the main focus of our analysis. In addition to the underdetermination entailed by the existence of multiple interpretations of a physical theory there is a subtly different class of underdetermination which grows out of the existence of multiple formulations. We can understand a difference in the formulation of a theory as different ways in which the theoretical (i.e., non-representative) structure of a theory can be expressed. More precisely, we can characterize the difference between two formulations in terms of a non-trivial difference as to the mathematical objects identified as the elements used to define both the state space and dynamical equations. ${ }^{3}$ Such a difference is realized in the paradigmatic cases of different formulations of the same theory: Lagrangian and Hamiltonian Newtonian mechanics. Here we have that the basic objects used to define

\footnotetext{
${ }^{2}$ Although there are some subtle issues there also, see (Janssen 1995, §3).

${ }^{3}$ These differences may also be found in cases of interpretational underdetermination, but are not on their own sufficient to generate such cases since they do not on their own entail difference in the representational roles played by the distinct formal elements.
} 
the dynamical equations and the states are non-trivially related since in the Lagrangian formulation we have positions and velocities (tangent vectors) and in the Hamiltonian formulation we have positions and momenta (cotangent vectors). This notion of what it is to be a different formulation is particularly useful since it precludes us from classifying different formulations simple 'notational variants' given by operations such as redefinition of co-ordinates. A crucial aspect of distinct formulations as opposed to distinct interpretations is that (as well as being confined to the non-representative aspect of the theory) they are necessarily accompanied with a rigorous translation dictionary which allows us to transform from the language of one formalism to the language of the other.

The interpretation and the formulation of a theory are closely related. A given interpretation may make use of a particular formulation of a theory and it may even be the case that a particular formulation is conducive to or exclusive of a particular interpretation. The strength of the relationship may not be particularly strong however: there will clearly be cases in which the various possible formulations of a theory are found to licence most, if not all, of the various interpretations equally. Nevertheless, in at least some important cases there is definite scope for the choice between competing formalisms to be restrictive enough to mandate only certain interpretations and therefore only certain ontologies. A important historical example of such a relationship between formulational and interpretational differences is that of the matrix mechanics and wave mechanics formulations of quantum theory when considered in the context of Schrödinger's (1926) equivalence proof 4. Although then considered as mathematically equivalent formulations of quantum theory, these were still taken by their supporters to imply distinct physical pictures of the world, in particular with regard to the treatment of space and time, and continuity (Bacciagaluppi and Valentini 2009, §4.6). Thus we see there is plausibility to the idea that underdetermination with regard to formulation can drive underdetermination with regard to interpretation $5^{5}$

The key to genuine cases of formulation underdetermination is the possibility of cases where two distinct formulations of the same theory place different bounds on the cast of viable interpretations. Clearly, trivially different formulations of a theory, such as notational variants, place no bounds upon the possible interpretations, and so cannot lead to any sense of ontological underdetermination. Non-trivially different formulations can of course also have this feature: although two formulations must reasonably be seen as distinct mathematical frameworks they may licence exactly members from a given cast of interpretations to exactly the same extent. For example, the difference between Lagrangian and Hamiltonian mechanics does not have any direct bearing upon

\footnotetext{
${ }^{4}$ Both the validity of this proof and, in fact, the equivalence of the supposed mathematically and empirically of the two formalisms at that time have been questioned (Muller 1997a; Muller 1997b). Thus our example should be taken more as an intuitive illustration than a concrete demonstration of the formulation difference/interpretation difference connection. Arguably at that time matrix mechanics and wave mechanics were simply different theories.

${ }^{5}$ Various authors have made arguments, on various grounds, towards one or another formulation of a given theory being more fundamental. This is true even for the most basic case of Lagrangian and Hamiltonian mechanics (North 2009, Curiel ming). Although worthy, such concerns are strictly tangential to the arguments of this paper since what is at issue here is whether there is a viable notion of ontology that a structural realist can recommend in the face of formulation underdetermination, and not with whether a traditional realist can 'break' underdetermination cases by finding means to privilege one formulation or another. See (French 2011) for further discussion of such issues.
} 
the interpretational dispute between 'relationalist' and 'substantivalist' regarding the interpretation of Newtonian mechanics in terms of a ontology with or without spacetime points ${ }^{6}$ However, on the other hand, the same formulational distinction does have implications for other interpretational disputes (see $§ 4.1$ ). Thus we see that formulational underdetermination is essentially sensitive to the interpretational context: whether or not a formulational difference is tied to an ontological difference depends entirely upon the particular type of interpretations we are working with.

We can distinguish three distinct notions of formalism underdetermination based upon three degrees of restriction that a difference in formulation may place upon the available interpretations (for a given interpretational context): First, the underdetermination may be strict, meaning that that there is no single interpretation that can be applied to both formalisms. Second, the underdetermination may be exclusive, meaning that there exists at least one interpretation which is applicable to one formulation but not to another. Third the underdetermination may be weak; meaning that a particular interpretation is more natural to one formulation than to another. All three variants are philosophically interesting since each (to a varying degree) leads to a situation whereby the nature of our ontology is dictated not by a choice between empirically consistent theories, nor even between interpretations of the same theory, but rather by the seemingly arbitrary choice between different formulations of the same theory.

What notion of realist ontology can be constituted in response to such cases of underdetermination? According to the proponents of ontic structural realism (OSR) the answer is a structural one. Whereas, traditional variants of object oriented realism (i.e., that based upon a distinct class of entities and things) must, in order to remain coherent, seek to break any genuine case of formulation underdetermination by appeal to some external criteria; by endorsing OSR one may side-step the undermining cut of underdetermination scenarios altogether 7 Under OSR, the representational capacity of a physical theory is provided by mathematical structures, that need not correspond to an ontology of objects and entities. Rather the ontology associated with our best scientific theories is one of concrete physical structures whose nature we can only understand via consideration of the abstract mathematical structures that are their linguistic correlates. Under OSR one may then seek to avoid formulation underdetermination entirely by limiting oneself to an ontology as represented by the structures common between two different formulations of a theory.

Notwithstanding the question as to whether the traditional realist responses of breaking underdetermination by appeal to external criteria may constitute a better alternative strategy, the onus is of course upon the proponent of OSR to be a little more specific about exactly what kind of mathematical structures they have in mind. In particular, it remains to be seen whether or not the specification of a structural ontology is on its own sufficient to resolve a genuine case of formulation driven ontological underdetermination (Pooley 2006). As is well illustrated by the alternative notion of 'structural realism' defended by North (2009) (her position is, within our terminology, a variant of conventional realism within which a geometric simplicity criterion is invoked in order to break formalism underdetermination), it is quite possible for the structural ontological vocabulary itself to be underdetermined if it is characterized in such a way

\footnotetext{
${ }^{6}$ There are, as ever, some subtleties here, see (Butterfield 2007, §2.3) for an extensive discussion.

${ }^{7}$ The conventional realist might of course alternatively simply dispute that genuine cases of underdetermination can every actually occur.
} 
as to be particular to each formalism. Thus, the structures that the defenders of OSR are looking to endorse must be such that they span between the appropriate formulations: it must be common structure. Pooley (2006, p.7) makes this point well in the context of the example of formulation underdetermination between wave and matrix mechanics mentioned above:

Through the work of Weyl and others, these formulations were soon recognized to be different representations of a single, mathematical structure in which states of a system correspond to rays in a Hilbert space, and observables correspond to operators (of the appropriate sort) that act on this space[...] A structural realist dissolution of [the] problem [of formulation underdetermination] requires an explicit characterisation of a mathematical framework that stands to each formalism as the abstract Hilbert space formalism of quantum mechanics stands to Schrödinger's wave mechanics and Heisenberg's matrix mechanics.

An obvious candidate for such structure is the mathematical transformations and interrelations that constitute the translation dictionary between two formulations. However, such a characterisation of the structural ontology is also problematic. As further noted by Pooley (2006), such interrelations between formulations offer only a very thin notion of structure that alone seems insufficient to be the fundamental furniture of the world: what is needed is a 'single, unifying framework [which we can] interpret as corresponding more faithfully to reality than do its various [conventional] realist representations' (p.7). Thus, the challenge to the ontic structural realist is to offer more than a purely set theoretic characterisation of the common underlying structure invoked to dissolve cases of underdetermination. What is needed, in essence, is a physico-mathematical framework that generalizes the structures relevant to each formulation in such a way as to illustrate that each formulation is merely a different representation of the same underlying 'reality'.

A further requirement, in a similar vein, is that the structural framework must reasonably be taken to include dynamical as well as purely mathematical aspects and must therefore be expected to be constituted by structures intimately connected to measurable, dynamical quantities. As noted by Bain (2009, p.22) such a requirement for 'dynamics encoding' structure would seem to point to a need to include more than simply group theoretic objects within our characterisation: 'to the extent that essential structure involves dynamics, we should perhaps be wary of identifying essential structure solely with group-theoretic invariants' 8 From the outset therefore, we are looking for a substantive notion of structure, fulfilling both Pooley's 'unifying framework' condition and Bain's 'dynamics encoding' condition, and we already have the indication that group theoretic structure alone will probably not be well suited to the job 9

\footnotetext{
${ }^{8}$ The cause of Bain's wariness is that in anti-self-dual Yang-Mills theories where '[t]he dynamics can be encoded not simply in the "invariants" of appropriate groups (i.e., in carriers of representations of such groups), but in spaces related to the carrying space of these groups.' (p.22) This case is well beyond the scope of the current paper, but we will briefly return to the question of dynamical structure needing more than groups in Section 4.3

${ }^{9}$ One might also, quite reasonably, insist that the structure to be reified is general enough to support all the empirical successes of the theory in question. According to Ruetsche (2011, §15.2.2) satisfaction of this requirement becomes problematic in realm of (infinite dimensional) relativistic
} 
There is thus a rather complex challenge to the proponent of ontic structural realism in balancing the need to find structures that are abstract enough to transcend particular formulations but concrete enough to be considered a genuinely dynamical unifying framework. The extent to which such a balance is achievable in practice is best evaluated through the consideration of a series of explicit examples, the simplest available of these will be presented in the final part of this paper. Before then, however, we must consider some important additional constraints on our principles for structural ontology construction which result from the interrelation of classical and quantum theories.

\section{Quantization and Structural Ontologies}

Quantization is a formal bridge between classical and quantum theories and thus provides a direct and rigorous way of linking historically successive theories. Formally, the quantization of a classical theory can be understood within a powerful and general geometric framework the essence of which is contained within the relationship between classical symplectic structure and quantum mechanical Hilbert space structure. Briefly put, a symplectic manifold is a smooth locally Euclidean space, $\mathcal{M}$, equipped with a closed non-degenerate two form, $\Omega$. A Hilbert space is an abstract vector space equipped with an inner product. Explicitly, under the geometric quantization programme (EcheverriaEnriquez et al. 1999) we seek a correspondence between the sets of pairs constituted by: symplectic manifolds $(\mathcal{M}, \Omega)$ together with smooth real functions $C^{\infty}(\mathcal{M})$, on the one hand; and complex Hilbert spaces, $\mathcal{H}$, together with self-adjoint operators $\mathcal{A}(\mathcal{H})$, on the other. We define the full quantization of a classical system $(\mathcal{M}, \Omega)$ as a pair $(\mathcal{H}, A)$ under certain conditions on $\mathcal{H}$ and the map, $A$, which takes us between classical and quantum observables. The conditions are:

1. $\mathcal{H}$ is a separable complex Hilbert space. The elements $|\psi\rangle \in \mathcal{H}$ are the quantum wavefunctions and the elements $|\psi\rangle_{\mathbb{C}} \in \mathbf{P} \mathcal{H}$ are the quantum states where $\mathbf{P} \mathcal{H}$ is the projective Hilbert space;

2. $A$ is a one to one map taking the classical observables $f \in C^{\infty}(\mathcal{M})$ to the self adjoint operators $A_{f}$ on $\mathcal{H}$ such that: i) $A_{f+g}=A_{f}+A_{g}$ ii) $A_{\lambda f}=\lambda A_{f} \forall \lambda \in \mathbb{C}$ iii) $A_{1}=I d_{\mathcal{H}}$;

3. $\left[A_{f}, A_{g}\right]=i \hbar A_{\{f, g\}}$ (i.e., $A$ is a Lie algebra morphism up to a factor);

4. For a complete set of classical observables $\left\{f_{j}\right\}, \mathcal{H}$ is irreducible under the action of the set $\left\{A_{f_{j}}\right\}$.

Of particular significance for our analysis is the third condition which encodes the relationship between the classical Poisson bracket and the quantum mechanical commutator. The former is defined implicitly by the symplectic structure $\Omega$, and thus the pivotal role of that structure in anchoring one end of the quantization bridge becomes apparent.

(n.b. There are a number of qualifications and extra subtleties regarding geometric quantization that might have been discussed in more detail. We have not, for example,

quantum theories. This paper will only focus upon non-relativistic 'ordinary' quantum theory, where such issues do not become relevant. Future work will consider Ruetche's problem in more detail. 
ventured into discussion of the Van Hove theorem, pre-quantization, polarisation, or other formal features that imply that symplectic structure is not on its own sufficient to guarantee a viable quantization. Although significant in and of themselves, such complications are not directly relevant to our investigation since the symplectic structure of the classical theory is still fundamental within more sophisticated understandings of the geometric quantization programme. See (Gotay 1980; Woodhouse 1997).)

That quantization itself is found to point to certain structures within the classical predecessor theory as in some way essential to the quantization of that theory is extremely interesting when considered in the context of ontic structural realism. One of the principal motivating arguments for the position (Ladyman 1998, p.420), is that it is observed that throughout the history of science empirically successful theories are often, if not always, replaced by theories which include starkly different types of theoretical entities and objects. From this we may make the pessimistically meta-inductive leap to the conclusion that the terms included within our current best theories that relate to theoretical entities and objects should not be thought of as constituting a genuine, robustly referential ontological vocabulary ${ }^{10}$ Rather, the proponents of OSR contend, we should focus our attention on the structural aspects of physical theory and attempt to reconceive the notion of what constitutes the ontological vocabulary in terms of the structure common between successive theories. If the formal structure of quantization techniques itself points to certain key structural facets of classical theory then it seems natural to ask what these structures correspond to within the quantum theory.

We may then be able to specify precisely the structures that, according to OSR, should be reified when constituting a structural scientific ontology spanning the classical/quantum boundary such that it is robust to the challenge of pessimistic metainduction. Clearly, the common structure motivated by such historical succession between theories may or may not be consistent with common structure between formulations discussed in the context of underdetermination above. Quantization provides us with exactly the mechanism to examine exactly such questions since it allows us to consider two different formulations of a classical theory and compare the relevant 'classical-classical' generalising structure, with any 'classical-quantum' common structure that can be identified 11 Furthermore, one would also hope to be able to find 'quantum-quantum' generalising structure. And then, if the OSR framework is a consistent one, one should be able to constitute a structural ontology that simultaneously transcends both inter-formational and inter-theoretic distinctions.

Here one might object that such a requirement for both diachronic and synchronic ontic structural continuity is unrealistically strong. If the world, at base, is 'really' a quantum one, then should not the proponent of OSR simply seek to uncover the

\footnotetext{
${ }^{10}$ There are, of course, more sophisticated varieties of scientific realism, such as that defended by Psillos (1999), that can be argued to circumvent the cut of the pessimistic meta-induction. Since our primary focus is upon examining the viability of OSR in of itself, rather than the strengths of its rivals we will neglect a full discussion of such nuanced versions of 'conventional' realism.

${ }^{11}$ An alternative structural approach to conceiving of an ontology at the classical/quantum boundary would be to focus upon the classical limit of the relevant quantum theories. We will here neglect a detailed consideration of this option since it would provide little insight into the inter-formulation issue which we wish to investigate in parallel. Similarly, we will also neglect consideration of theories, such as thermodynamics and fluid mechanics, without natural quantum correlates. Although, in such contexts, quantization probably can no longer serve as a guide to ontic structure, it would still be hoped that consideration of the structure of the predecessor-successor relationship would.
} 
fundamental quantum structure, and neglect earlier classical theories altogether. There are several lines of response to such an objection. First, if we follow Ladyman in seeing the challenge of pessimistic meta-induction as a motivation for OSR, then it is incumbent upon us to require the ontology established by the position to be of a type resistant to the argument. Since we do not have access to future physical theories, the only way to find a methodology for constructing structural ontologies resistant to the pessimistic meta-induction surely must be to look to the past, as advocated. Second, and more specifically, although it is true that as far as electromagnetic and nuclear interactions go our current best theories are quantum mechanical, it still remains to be seen whether gravitation can be quantized consistently. Thus, at the moment, considering the structure behind 'our best theories of physics' must entail considering both classical and quantum theories. Thirdly, and more constructively, although one might not expect it, the process of quantization actually does prove a guide to finding at least some of the basic structure fundamental to quantum theories. This will be illustrated explicitly in $\S 4.3$. However, that is not to say that it is the only guide, or that purely quantum structure is uninteresting. Rather, this project is predicated upon the intuition that by understanding diachronic structural relationships between quantum theories and their classical predecessors, we will get insight into what type of quantum structures will survive into the future successor theories.

Let us now try and formalize the idea of dual diachronic/synchronic ontic structural continuity more explicitly. Let us assume we are given two formulations of a classical theory $C_{1}, C_{2}$. A genuine implementation of the OSR programme for resolving cases of underdetermination should then provide us with a generalising framework for the pair of classical formulations, $C_{G F}$. Similarly, given two formulations of the corresponding quantum theory, $Q_{1}, Q_{2}$, a genuine implementation of the OSR programme should allow us to define a quantum mechanical generalising framework, $Q_{G F}$. The structural basis for quantization should then provide us with a guide towards further generalising the two frameworks such that an even more general framework, connecting the classical and quantum structures, can be established. This doubly generalizing framework, $C Q_{G F}$, will, by construction, be immune to both pessimistic meta-induction arguments and formulation underdetermination arguments. We can illustrate the relationship between the various formalisms and structural generalisations as show in Diagram 1.

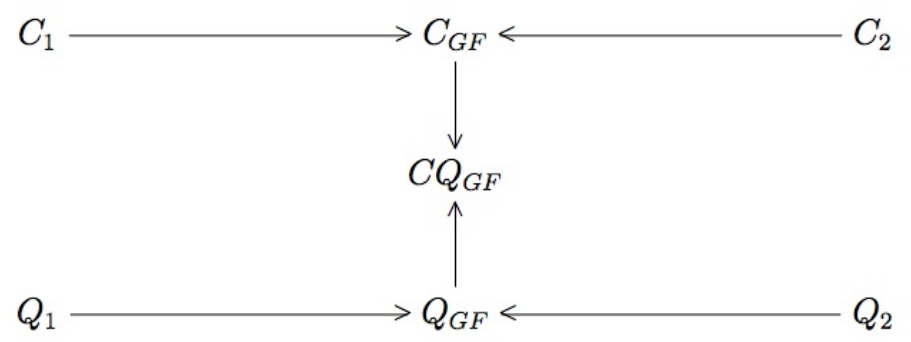

Diagram 1. Representation of the relationship between the various formalisms and structural generalisations for a classical theory and its quantum analogue.

Armed with mathematically well defined quantization procedures and interrelations between formulations we may perhaps be able to make progress in implementing this schema by considering a test case. The most basic case available is that of classical/quantum nonrelativistic particle mechanics, and it is to this that we now turn. 


\section{The Case of Non-Relativistic Particle Mechanics}

\subsection{Formulation Underdetermination}

Let us consider a classical system consisting of a finite number of degrees of freedom and assume that this system does not contain any local symmetries ${ }^{12}$ The physical theory describing such a system is Newtonian mechanics and in modern terms the two principal formulations available are Lagrangian and Hamiltonian 13 The Lagrangian formulation of Newtonian mechanics is framed within the space of solutions to the Euler-Lagrange equations which are dynamical curves, $\gamma_{E L}: T \mathcal{C} \rightarrow \mathbb{R}$, in the velocity-configuration space (the tangent bundle to configuration space), $\left(q_{i}, \dot{q}_{i}\right) \in T \mathcal{C}$. The Hamiltonian formulation of Newtonian mechanics is framed within phase space (a symplectic manifold defined as the cotangent bundle to configuration space), $\left(q_{i}, p_{i}\right) \in T^{\star} \mathcal{C}$, with Hamilton's equations, $\Omega\left(X_{H}, \cdot\right)=\mathbf{d} H$, picking out a preferred tangent vector field on phase space, $X_{H}$, which is sufficient to define the set of dynamical curves for any specification of instantaneous initial data.

By the criteria and definitions detailed above what we are dealing with here is two distinct formulations of the Newtonian theory of mechanics: neither Lagrangian nor Hamiltonian formalism furnishes us with an ontology without a further interpretation; the basic equations and state spaces of each formalism are defined in terms of different basic mathematical objects; and the two are connected by a rigorous translation dictionary provided by the Legendre transformation together with the set of maps (parameterized by a one dimensional time parameter) that exists between a given solution in the Lagrangian formulation and the corresponding sequence of instantaneous states in the Hamiltonian formulations. The crucial question, in light of our above analysis, is then whether we should understand these formulations as leading to an underdetermination of the relevant ontology. This depends on the nature of the relevant interpretations available and their relationship to these two formulations.

Focusing in particular on the temporal ontology of Newtonian mechanics, two candidate interpretations are available. The first is constituted by the classic Newtonian picture of instantaneous states of the world together with deterministic laws sufficient to fix all past and future states given an initial state. We will call this the instantaneous picture of the world and understand it as specifying an ontology which posits instantaneous states as part of the fundamental furniture of the world. Supplementary to this picture we can ascribe additional and more metaphysical structures such as a dynamical notion of time and an ontologically privileged present (Markosian 2011). Our concern here is not with the detailed philosophical analysis of these additional interpretational structures and the extent to which they prove acceptable additions to the project of furnishing the relevant theory with an ontology. They are certainly not generally taken to be precluded by Newtonian mechanics at least ${ }^{14}$ Rather, what we shall assume to be at the very least non-controversial is that given the viability of an interpretation in terms of an instantaneous picture, one may, if it is deemed reasonable, supplement this interpretation with additional temporal ontic structure such as a dynamic time.

\footnotetext{
${ }^{12}$ Here and below we neglect the role of global symmetries for the sake of brevity. An analysis of the structural connections relevant to them would follow straightforwardly from what we say about observables and state spaces.

${ }^{13}$ Unfortunately, we do not here have space to consider the Hamilton-Jacobi formulation also. See (Butterfield 2005) for a discussion of the relationship between that formalism and quantum theory. The expansion of the classical structural framework below to include HJ theory would appear fairly natural given the central role that symplectic structure plays within that formalism.

${ }^{14}$ See (Wüthrich 2010) for an interesting discussion of the extent to which the presentist view is precluded by theories of quantum gravity of exactly the type that have been extensively detailed in this work.
} 
A second interpretation of Newtonian mechanics that provides us with a distinct temporal structure is in terms of entire four dimensional histories which are specified by atemporal laws (i.e., laws that are not defined at a given time) together with initial and final boundary conditions. We will call this the teleological picture of the world since it implies the final boundary data is fundamental in determining the laws. Unlike the instantaneous picture it does not posit instantaneous states as part of the fundamental furniture of the world and, relatedly, it is not as amenable to supplementation with the additional more metaphysical structure mentioned above and discussed in more detail below. We do not mean this as necessarily a particularly strong claim and will not therefore seek to make a justification of it in a strong sense. Rather, we believe it is at the very least reasonable to assume that an interpretation of Newtonian mechanics in terms of a teleological picture is, at face value, going to look more like a non-dynamic, 'eternalist' type stance as to the metaphysics of time and less like the dynamic/privileged present type stances ${ }^{15}$

Just as there is a clear intuitive relationship between the aspects essential to the instantaneous picture and the presentist stance, there is a clear intuitive relationship between the aspects essential to the teleological picture and the anti-presentist stance. It would seem, furthermore, that the teleological picture is such that it is inherently hostile to presentism: the laws, boundary conditions and fundamental objects are things that, by the presentist lights, do not exist. Thus, at a superficial level of analysis at least, there is a natural way of cashing out the difference between our two pictures in terms of a substantive ontological difference 16

Even if we were to be more minimalist as to the level of metaphysical structure we wish to permit, then we may still end up with genuine differences between the two pictures. Whereas the instantaneous picture is predicated upon an ontology that necessarily includes instantaneous states as fundamental, the teleological picture is not necessarily predicated upon such an ontology. Thus any approach to space-time ontology which precludes fundamental instantaneous states can only be reconciled with the teleological picture, and is thus more naturally at home within the Lagrangian formalism. Such an argument is of course not sufficient to establish that there is no reasonable conventional realist ontology that transcends the Lagrangian/Hamiltonian divide. Rather, we have that there is at least some notion of ontology that are underdetermined by the case in hand, and thus that there is a requirement for the proponent of OSR to provide a viable alternative ontology.

Let us then proceed to examine our case in terms of our framework for formalism underdetermination. We have two formulations of a theory together with two viable and distinct interpretations (or interpretation types). Above we listed three ways in which such a situation may lead to formalism underdetermination: the underdetermination may be strict, meaning that there is no single interpretation that can be applied to both formalisms; it may be exclusive, meaning that there exists at least one interpretation which is applicable to one formulation but not to another; and it may be weak, meaning that one or more of the interpretations are more natural to one formulation than to another.

Since the teleological interpretation (or interpretation type) is applicable to both Hamiltonian and Lagrangian formulations the first does not apply. One could argue for the second

\footnotetext{
${ }^{15} \mathrm{~A}$ different, more exotic, interpretational option for the temporal ontology of Newtonian mechanics is a 'gunky' or 'pointless' temporal topology, see (Arntzenius 2011). Applying such a formalism/interpretation package would appear to entirely rule out both the instantaneous picture and an ontologically privileged present.

${ }^{16}$ There is also reasonable scope to understand the difference between the instantaneous and teleological pictures as possibly grounding a fundamental metaphysical difference as to the laws of nature. For example, it has been claimed that the disposition essentialist viewpoint on laws of nature is inconsistent with the principle of least action that is fundamental to the teleological picture (Katzav 2004). See $($ Smart 2012, §8) and (Thebault and Smart 2013) for further discussion.
} 
on the grounds that the instantaneous interpretation might seem not to be applicable to the Lagrangian formulation. However, one may reconstruct the Lagrangian formulation such that it is based upon points rather than curves within the tangent bundle and such that the dynamical equations are differential equations giving a unique specification of dynamics at such a point rather than restrictions on possible curves. Such a re-conception means that it is possible to apply an instantaneous interpretation to the Lagrangian formalism. However, the historically prior and arguably most fundamental way of understanding Lagrangian mechanics is in the context of action principles and variational calculus and such formal structure does necessarily lead to a formulation which is in terms of curves with two boundary conditions. There is therefore a good case for the Lagrangian formulation being more naturally interpreted in teleological rather than instantaneous terms and thus for us being confronted with a weak case of formalism underdetermination.

Given that the solution space of the Lagrangian formulation is that of curves with two boundary conditions, the natural interpretation is one in terms of a histories based ontology; with the furniture of the universe entire four dimensional spacetimes along with the appropriate initial and final conditions (i.e., the teleological picture). Given that the solution space of the Hamiltonian formulation is an initial data space, the natural interpretation is in terms of an instantaneous state based ontology; with the furniture of the universe three dimensional spatial states with appropriate instantaneous data (i.e., the instantaneous picture). Since the two formulations are empirically equivalent and yet, to an extent, furnish us with distinct ontologies we have a good case within which to examine the problem of constructing a viable structural ontology.

Clearly, although a positive result would not be sufficient to demonstrate superiority of OSR over its conventional realist rivals, since we have not yet convincingly proved a case for the underdetermination of their ontology, a negative result would serve to severely weaken the tenability of the OSR position: if one cannot find a consistent structuralist ontology for the simplest of cases then the prospects for the programme to find wider application look grim.

\subsection{The Classical Ontology}

The ontic structural realist response to cases of formalism underdetermination is to seek to reconceive the relevant notion of ontology in structural terms such that it is no longer underdetermined. For such structure to genuinely constitute an ontology it is required to consist of more than a mere interrelation between formulations, we need to find a suitably generalising physico-mathematical framework which includes the requisite level of dynamical structure. Is this possible for the case of Lagrangian and Hamiltonian mechanics?

Following the analysis of Belot (2007) and the arguments of French (2011), we can make a good argument that the answer is yes. For all standard theories of classical mechanics, the space which represents unique solutions within a Lagrangian formulation of mechanics, has a close formal relationship with the space which uniquely represents instantaneous states within a Hamiltonian formulation. Within Newtonian mechanics these two spaces are simply the space of curves solving the Euler-Lagrange equation, $\gamma_{E L} \in \mathcal{S}$, and phase space, $T^{\star} \mathcal{C}$. Not only are these two spaces connected by a set of maps between time slices of Lagrangian solutions and instantaneous canonical states, but since $\mathcal{S}$ is, like phase space, a symplectic manifold, it is possible to prove that the two relevant dynamical arenas are symplectically isomorphic. The existence of this symplectic isomorphism then allows us to fix a precise relationship both between functions representing observable quantities within the two formalisms and between the representation of dynamical change in the observable quantities. Given a preferred slicing of a Lagrangian solution, for every moment of time we can construct a symplectic isomorphism between a phase space function and a corresponding function on $\mathcal{S}$, and this relationship allows 
us to understand both functions as representing the same underlying physical quantity as it changes over a dynamical history.

Thus the mutually symplectic structure of Lagrangian and Hamiltonian mechanics provides us with the key towards isolating exactly the kind of generalising framework, including dynamical structure, which we are looking for and, although we will certainly not claim that this analysis is complete, there is a convincing case for an ontic structural realist account of the Hamiltonian and Lagrangian formulations of Newtonian mechanics in symplectic terms. This ontology is not constituted by the symplectic isomorphism itself but by the generalized notion of symplectic state space and observables algebra that stands behind the individual Lagrangian and Hamiltonian realisations. Thus the interconnections between dynamical structures that the isomorphism encodes, at the level of both observables and the state spaces, can be understood as manifestation of the deeper structural ontology of mechanics. To accept this ontology is not to endorse either the instantaneous or teleological interpretations, rather through OSR we are able conceive of a fundamental reality that stands behind these two contrasting pictures of the world.

The question still remains: how should we understand the correspondence between this structural ontology described in terms of abstract mathematical objects and physical structures in the world? Most importantly, given the connection to the relevant empirical measurements comes via the entire set of observables, how should we understand the status of modal structure within our ontology? We will return to this issue in in $\S 4.4$, after we have considered the extension of our program into the quantum domain.

\subsection{Quantum Theory and the Generalized Structural Ontol- ogy}

The basic formalism of a quantum theory is given by the combination of a complex Hilbert spaces, $\mathcal{H}$, together with self-adjoint operators, $A \in \mathcal{A}(\mathcal{H})$. Such mathematical structures provide a simple example of the type of (largely) interpretation independent formalism. Furthermore, there exist precise criteria for establishing when two different quantum formalisms are equivalent. Specifically we require that: i) the two Hilbert spaces can be shown to be unitary isomorphic; and ii) the relevant isomorphism can be understood as entwining the representations of two sets of quantum observables. This is of course very similar to the picture we arrived at for classical theory, with the part previously played by the symplectic isomorphism now played by a unitary isomorphism, and thus suggest a degree of coherence in our approach. We then have a simple and elegant means to characterize a notion of quantum structural ontology, $Q_{G F}$, in terms of a generalized notion of Hilbert space and observables algebra both of which stand behind the various realisations, which are connected in terms of i) and ii). Such a candidate for a quantum structural ontology clearly fulfils the Pooley 'generalising framework' criterion, does it also satisfy the Bain 'dynamical structure' requirement? How is dynamics encoded in the Hilbert space plus observables package? As in the classical case, evolution of observables is defined by the action of the Lie bracket (in this case the commutator) combined with the Hamiltonian observable, i.e., $\dot{A}_{f}=i \hbar\left[A_{f}, A_{H}\right]$ for an arbitrary observable $A_{f}$ and the Hamiltonian operator $A_{H}$. With regard to the state space itself we also have a notion of dynamics in terms of the the $U(1)$ temporal automorphisms on Hilbert space generated by the Hamiltonian operator. Thus, we see that the dynamics is encoded in a partially group-theoretical manner, but, as emphasized by Bain in the quote above, can only be fully understood by reference to the relevant algebraic and geometric structures - i.e., the inner product structure of the state space or the Lie algebra structure of the observables.

We then come to the question most crucial to our analysis. Is this prospective structural 
ontology we have been sketching of the suitable type to deal with both formulation underdetermination issues and the historical undermining of pessimistic meta-induction? In the first instance, we would like to know if it is appropriate to conceive of common structure spanning between the generalized classical and quantum mechanical framework?

At first sight the evidence appears limited: whereas the generalized notion of a classical state space is given by a manifold with symplectic structure, the analogous object in a generalized quantum mechanical context, the Hilbert space, is a linear vector space not based upon a symplectic geometric structure. However, as was seen in our discussion of geometric quantization techniques in $\S 3$, if we focus on the observables algebra relevant to a a generalized notion of quantum theory (i.e. $Q_{G F}$ ) then we do find a quantum mechanical analogue to the Poisson bracket (which is of course defined by the symplectic structure). As discussed above, one of the key steps in geometric quantization is the definition of the map $A: f \rightarrow A_{f}$ which takes us between the classical algebra of observables, defined by functions on a symplectic manifold, and the quantum algebra of observables, defined by self-adjoint operators on a Hilbert space. One of the restrictions on this map is that:

$$
\left[A_{f}, A_{g}\right]=i \hbar A_{\{f, g\}}
$$

and thus we see that by definition the geometric quantization scheme is such that the classical Poisson bracket structure is carried over into the quantum context in terms of the commutator. We can therefore justifiably argue that there exists a structural bridge between the observables of classical Newtonian mechanics and non-relativistic quantum mechanics at a formal level, precisely in terms of the link between the binary operations constituted by the Poisson bracket and the commutator. This analogy is also reflected at the level of dynamics since when combined with the Hamiltonian observable it is the binary operation that is responsible for generating evolution in both the classical and quantum realms - i.e., we have that $\dot{A}_{f}=$ $i \hbar\left[A_{f}, A_{H}\right]$ and $\dot{f}=\{f, H\}$. Of course the Poisson bracket is itself defined implicitly by the classical symplectic structure, $\Omega$, and thus we have that it is the symplectic structure of the algebra of classical observables that provides the foundations of the quantum mechanical algebra.

Independently of anything to do with formalism underdetermination, a proponent of OSR would therefore argue that the fundamental structure of a classical or quantum theory is related to maps between algebras of observables and the relevant binary operations. Fundamentally this is what is structurally consistent between the classical and quantum theories. It is therefore precisely what the structural framework we are looking for should serve to generalize in order to constitute an ontology immune to pessimistic meta-inductive arguments. However, this is also the type of structure which we were driven towards when considering the ontology of the classical theory alone so there would seem to be prima facie coherence in our approach.

Given our two classical formulations of particle mechanics we arrived at a structural ontology constituted by a generalized notion of state space and observables algebra. This we established on the basis of the symplectic isomorphism connecting the observables and state spaces of Lagrangian and Hamiltonian formulations: it is precisely this structural relationship that warrants the interpretation of the two formalisms as constituting two possible realisations of an underlying, more general framework for classical particle mechanics. A symplectic isomorphism is a type of Lie algebra morphism and so there is consistency between the relevant homomorphism in each case. This gives credence to the idea generalized package of 'state space plus observables algebra' which we could understand as underlying various realisations within both classical and quantum theories of particle mechanics. But as ever, the devil is in the details. The central question is whether such classical-quantum structural connections can serve as a guide to allows us to construct a concrete classical-quantum structural framework 
that fulfils the requirements discussed above of being simultaneously generalising (in the relevant sense), substantial and dynamical. Most importantly, although the discussion above has allowed us to find a close formal connection between the relevant observable algebras, we have, as yet, not said very much about the state spaces and the binary operations which they are equipped with. In the remains of this section we will examine a relatively under-appreciated geometric formulation of quantum theory that allows us to explicitly make these connections, and, in doing so, identify a candidate for our fully generalising structural ontology.

First, let us view the Hilbert space of a quantum theory as a real vector space equipped with a complex structure $J{ }^{17}$ This complex structure is a linear mapping $J: \mathcal{H} \rightarrow \mathcal{H}$ such that $J^{2}=-1$. If we now consider two states $|\psi\rangle,|\phi\rangle \in \mathcal{H}$, then we may decompose the inner product as:

$$
\langle\psi \mid \phi\rangle=\frac{1}{2 \hbar} G(\psi, \phi)+\frac{i}{2 \hbar} \Omega(\psi, \phi)
$$

where $G$ is a positive definite, non-degenerate, real inner product and $\Omega$ is a closed, strongly non-degenerate two form. We can thus identify $G$ as a Riemannian metric and $\Omega$ as a strong symplectic two form 18 The metric and symplectic, and complex structures are related straight forwardly as:

$$
G(\psi, \phi)=\Omega(\psi, J \phi)
$$

and this implies that the triple $(J, G, \Omega)$ equip $\mathcal{H}$ with the structure of a Kähler Space.

Crucially, we can then make the canonical identification of the tangent space to any point in the Hilbert space, $T_{\psi} \mathcal{H}$, with $\mathcal{H}$ itself ${ }^{19}$ Given this, $\Omega$ is naturally extended to a (strongly) non-degenerate closed differential two-form on $\mathcal{H}$. Any Hilbert space can thus be identified as a (strong) symplectic manifold. This means that, as in the classical case, the generalized notion of state space which is relevant to quantum theory is one equipped with a Poisson bracket structure. What we do not yet have, however, is a connection between the quantum Lie bracket that is relevant to the quantum algebra of observables, and the Poisson bracket that is relevant to the quantum state space. In the classical case the relevant Lie bracket simply was the relevant Poisson bracket, and for our generalized structural framework to be consistent it would seem necessary for there to be something like such a connection in the quantum arena.

To see that this is indeed the case, let us first define the expectation value function, $\langle A\rangle_{\psi}$ : $\mathcal{H} \rightarrow \mathbb{R}$, via:

$$
\langle A\rangle_{\psi}:=\langle\psi, A \psi\rangle=\frac{1}{2 \hbar} G(\psi, A \psi)
$$

it can then be demonstrated that:

$$
\left\{\langle A\rangle_{\psi},\langle B\rangle_{\psi}\right\}=\left\langle\frac{1}{i \hbar}[A, B]\right\rangle_{\psi}
$$

${ }^{17}$ Here we mainly follow (Ashtekar and Schilling 1999), but also see (Boya et al. 1991), (Marsden and Ratiu 1995, §5), and (Corichi 2008).

${ }^{18}$ It is essential here that the two from is not merely a weakly non-degenerate symplectic form since in that case the definition of the inner product no longer holds. See (Abraham and Marsden 1978, p.173)

${ }^{19}$ This is because the abelian transformation group allows us to define the relevant isomorphism by associating with each $\psi \in \mathcal{H}$, the tangent vector $\dot{\psi} \in T_{\psi} \mathcal{H}$ given by

$$
\dot{\psi} f(\phi):=\left.\frac{d}{d t}\right|_{t=0} f(\phi+t \psi)
$$

give any $f \in C^{\infty}(\mathcal{H})$. (Boya, Cariñena, and Gracia-Bondía 1991). 
where $A$ and $B$ are quantum observables; $\langle A\rangle$ and $\langle B\rangle$ are expectation values; $\{$,$\} is the$ quantum Poisson bracket; and [,] is the commutator. The resemblance to classical-quantum algebraic correspondence equation (1) above is of course striking. However, this is strictly a quantum equation telling us that expectation value of the commutator between two observables is given by the quantum Poisson bracket of the operator's expectation values. Thus, as in the classical case, there is a deep mathematical connection between the Lie bracket structures relevant to algebra of observables and the Poisson bracket structures relevant to the state space 20

We are now in a position to make a precise specification of the structural framework, built upon common structure, that generalizes classical and quantum mechanics in a suitable sense. Following the notation introduced earlier, we already have that the classical generalising framework, $C_{G F}$, is given by the duple: $(\mathcal{M} ; \mathfrak{F})$, where $\mathcal{M}$ is a symplectic manifold, smooth functions upon which define an algebra of observables, $\mathfrak{F}$, which is a Lie algebra with the symplectic form defining the relevant Lie bracket as the Poisson bracket. The quantum generalising framework is simply given by the duple: $(\mathcal{H}, \mathfrak{A})$, where $\mathcal{H}$ is a Hilbert space, and the algebra of $\mathfrak{A}$ is again a Lie algebra, and is given by self-adjoint operators on the Hilbert space, with the commutator defining the Lie bracket. The idea is then, given the formal connections we have just presented, to define $C Q_{G F}$ as a duple: $(\mathcal{P}, \mathfrak{L})$, where $\mathcal{P}$ is a general notion of state space as a symplectic manifold (with accompanying Poisson bracket), and $\mathfrak{L}$ is a general notion of algebra of observables as a Lie algebra. This framework viably generalizes classical and quantum particle mechanics and makes essential use of dynamical structure. It also isolates precisely the mathematical structure that is common to both classical and quantum mechanics, whilst simultaneously showing how we can understand key aspects of the difference between the two in terms of a difference in the relationship between the observables Lie bracket and state space Poisson bracket in each case 21

A proponent of OSR would therefore have good grounds to argue that the fundamental structure of classical and quantum particle mechanics is constituted by the general mechanical framework $(\mathcal{P}, \mathfrak{L})$, standing as it does behind the individual classical and quantum formalisms. The viability of such a generalisation of particle mechanics is built upon: 1) the classicalclassical and classical-quantum algebraic relationships defined via Lie algebra morphisms; 2) the mutually fundamental role of the Poisson brackets found within classical and quantum state spaces; 3) The connections between the Lie bracket relevant to the algebras of observables and the Poisson bracket relevant to the state spaces. This constitutes a proof of principle for our program for implementing ontic structural realism via simultaneous diachronic and synchronic formulation generalisations. What interpretation should we give to these results?

${ }^{20}$ We should note here that there is a good basis to consider the projective Hilbert space, $\mathbf{P} \mathcal{H}$, as the quantum state space rather than simply $\mathcal{H}$ itself. Here we avoid making this refinement of the formalism due to a desire to restrict the amount of technical material presented. The reader is directed to (Ashtekar and Schilling 1999) §B.) where it is shown that $\mathbf{P} \mathcal{H}$ is also naturally equipped with symplectic structure, and that the connections between the state space Poisson bracket and observables algebra Lie bracket structure is in fact even stronger.

${ }^{21}$ In particular, it is important to note that the metric $G$ is not rooted in any structure within the classical formalism, but clearly does have an important physical role in quantum theory (see Ashtekar and Schilling 1999) §IIA 2.). $G$ is, however, implicitly encoded within $C Q_{G F}$ since, through Equations (5) and (6), it dictates the particular sense in which the general Lie bracket and state space Poisson bracket relationship is embedded in the particular quantum case. 


\subsection{Interpretation of Results}

The first and most important implication of the foregoing analysis is that both Pooley's 'unifying framework' condition and Bain's 'dynamics encoding' condition, can be satisfied by a proponent of OSR, confronted with the task of defining the generalising structure behind both diachronically and synchronically related physical formalisms. This is a non-trivial result and provides evidence for the coherence, if not supremacy, of structuralist approaches to characterising the ontology of science.

A second point which our case study serves to illustrate is how the process of establishing substantive structural ontologies could be taken to proceed in practice. We first identified the shared structures between the various formalisms of a theory by isolating the relevant homomorphisms (this would seem to be in keeping with the analysis given in (Landry 2007)). We then proceeded to consider a generalized version of these structures, understood as standing behind the various realisations and underpinning the structural relationships. We then repeated the process for a theory which is historically related to the other (either as a successor or predecessor). We then sought to evaluate potential structural relationships between the diachronically related generalized structures in order to establish an even more general structural framework standing behind both synchronically and diachronically related formalism. The process of quantization proved instrumental to our investigation precisely because it provided a precise methodology for making manifest the diachronic structural relationships relevant to the case at hand. In this case at least, such a constructive methodology leads naturally towards an algebraic and geometric characterisations of structure, rather than a construction purely in terms of group or set theoretic based notions. In general it seems more reasonable for us to expect the details of the physical theories themselves (and the relationship between them) to serve as a guide to the fundamental structure of physical theory, rather than assuming a particular mathematico-logical formalism from the outset, and attempting to shoehorn physics into it.

A third, more metaphysically oriented, point is that the connection between the structures that we end up reifying within the approach presented, and the 'physical structures in the world', has implications for the status of modal structure within OSR. By investing in an ontology built around an abstract notion of state space and algebra of observables, we do not strip ourselves of the ability to talk about physical structures in the world (and fall into an extreme form of Platonism) precisely because of the links between measurements of empirical quantities and the the observables (or expectation values). However, since we take the entire algebra and state space together, the framework does not ontologically privilege the empirical quantities determined by the initial conditions of the world we actually live, all possible empirical quantities have equal status. This might be taken to constitute precisely the ontological commitment to modal structure that, according to Ladyman and Ross (2007), is the key feature distinguishing OSR from the structural empiricism of van Fraassen (2006, 2008) .

Thus on one reading, our approach, which was intended from the outset to be both metaphysically sparse and closely grounded in science, appears to end up entailing strong ontological commitments with regard to modality. This might be seen to cast doubt upon the extent to which the principles of naturalism are being respected within the approach (see (Berenstain and Ladyman 2012) for further discussion of this and related points).

Alternatively, one might rather insist that the structural framework articulated here would only ever be partially instantiated, thus only particular values of the observables, and particular regions of the state spaces are ever genuinely realized. This, however, would seem to blur the lines between ontological structural realism, on the one hand, and structural empiricism and epistemic structural realism, on the other. Our commitment would be to fundamentally 
hidden ontology as constituted by the (structured) algebra of all possible observations within a single universe, but we would admit epistemic access solely to the unrestricted state-space structure describing all possible universes. Such a position seems rather strange, but warrants further investigation.

\section{Conclusion and Prospectus}

Based upon the analysis of Section $\S 4$ it seems reasonable to conclude the following: A general mathematical framework can be provided for the fundamental architecture of classical and quantum non-relativistic particle mechanics. On our reading, OSR implies that such a framework should be used towards the establishment of a structural ontology for physical theory in terms of an abstract notion of state space and algebra of observables. Given such a specification, one has an ontology general enough to avoid both the problems of pessimistic meta-induction and formalism underdetermination, but concrete enough to provide a substantive, dynamical and empirically grounded characterisation of reality. Endorsing such a framework can be seen to have non-trivial implications for the ontological status of modal structure.

The key question is then to what extent do the results of our investigation of nonrelativistic particle mechanics offer us hope for further generalisation? Is it reasonable to expect our success to be repeatable in the more fundamental and more complex realm of classical and quantum field theories? Classically at least, there are good reasons to be optimistic: although they are formally far more complicated, classical field theories (including general relativity) are still essentially reducible to states spaces with symplectic structure together with Poisson bracket algebras of observables. In the field theoretic context, however, quantization becomes an almost entirely different animal. Even putting aside the infamous problem of quantizing general relativity, when one considers field theories one finds that, formally speaking, the various paths towards the quantization of the same theory seldom lead to even approximately the same destination. Yet, one may still expect that crucial aspects of our methodology will be relevant in that context. For instance, as noted in (Thébault 2012), that one fails to find an observable entwining isomorphism between two Hilbert spaces derived via different quantization methodologies, need not be taken to imply that no philosophically significant structural relationship between the relevant state spaces and observables can be isolated. The treatment of gauge groups and degrees of freedom, in particular, allows us to establish a connection between the observable algebras and states spaces of different quantum formulations of the same field theory, even when a formal mapping cannot be established. Thus there are good reasons of principle, which would be hoped to be born out in practice, to expect our approach of using quantization as a guide to ontic structure, to be applicable to a second, field theoretic case study ${ }^{22}$

\section{Acknowledgements}

Thanks to Sam Baron, Radin Dardashti, Richard Dawid, Pete Evans, Stephan Hartmann, Sebastian Lutz, Hannes Leitgeb, Thomas Meier, Oliver Pooley, Huw Price, Dean Rickles, two anonymous referees, and audiences in Sydney, Munich and Hannover for valuable comments on various versions of this material. Research for this article was supported initially by the Centre

\footnotetext{
${ }^{22}$ In this vein, it is anticipated that the further case study will find connections with the work of Ruetsche (2011) regarding characterisations of field theoretic structure in terms of algebras of observables
} 
for Time (University of Sydney) and, more recently, by the Alexander von Humboldt Foundation and the Munich Center for Mathematical Philosophy (Ludwig-Maximilians-Universität München).

\section{References}

Abraham, R. and J. E. Marsden (1978). Foundations of Mechanics. San Francisco: Benjamin/Cummings.

Arntzenius, F. (2011). 'Gunk, topology and measure'. In Logic, Mathematics, Philosophy, pp. 327-43. New York: Springer.

Ashtekar, A. and T. A. Schilling (1999). 'Geometrical formulation of quantum mechanics'. In On Einstein's Path, pp. 23-65. New York: Springer.

Bacciagaluppi, G. and A. Valentini (2009). Quantum Theory at the Crossroads: Reconsidering the 1927 Solvay Conference. Cambridge: Cambridge University Press.

Bain, J. (2009). 'Towards structural realism'. <ls.poly.edu/ jbain/papers/SR.pdf >.

Belot, G. (2007). 'The representation of time and change in mechanics'. In J. Butterfield and J. Earman (Eds.), Handbook of Philosophy of Physics, pp. 133-227. Amsterdam: North-Holland.

Berenstain, N. and J. Ladyman (2012). 'Ontic Structural Realism and Modality'. In D. Rickles and E. Landry (Eds.), Structural Realism: Structure, Object, and Causality, pp. 149-68. New York: Springer.

Boya, L. J., J. Cariñena, and J. Gracia-Bondía (1991). 'Symplectic structure of the Aharonov-Anandan geometric phase'. Physics Letters A 161(1), 30-4.

Butterfield, J. (2005). 'On Hamilton-Jacobi theory as a classical root of quantum theory'. In A. C. Elitzur, S. Dolev, and N. Kolenda (Eds.), Quo vadis quantum mechanics?, pp. 239-73. Berlin: Springer.

Butterfield, J. (2007). 'On Symplectic Reduction in Classical Mechanics'. In J. Butterfield and J. Earman (Eds.), Philosophy of Physics, pp. 1-131. Amsterdam: North-Holland.

Corichi, A. (2008). 'On the geometry of quantum constrained systems'. Classical and Quantum Gravity 25(13), 135013.

Curiel, E. (forthcoming). 'Classical mechanics is Lagrangian; it is not Hamiltonian'. The British Journal for the Philosophy of Science.

Echeverria-Enriquez, A., M. Munoz-Lecanda, N. Roman-Roy, and C. Victoria-Monge (1999). 'Mathematical foundations of geometric quantization', < arxiv.org/abs/math$\mathrm{ph} / 9904008>$.

French, S. (2011). 'Metaphysical underdetermination: why worry?'. Synthese $\mathbf{1 8 0}(2), 205-$ 21.

French, S. and J. Ladyman (2003). 'Remodelling Structural Realism: Quantum Physics and the Metaphysics of Structure'. Synthese 136, 31-56.

Frigg, R. and I. Votsis (2011). 'Everything you always wanted to know about structural realism but were afraid to ask'. European Journal for Philosophy of Science 1(2), 22776.

Gotay, M. J. (1980). 'Functorial geometric quantization and Van Hove's theorem'. International Journal of Theoretical Physics 19(2), 139-61. 
Janssen, M. H. P. (1995). A comparison between Lorentz's ether theory and special relativity in the light of the experiments of Trouton and Noble. Ph. D. thesis, University of Pittsburgh.

Jones, R. (1991). 'Realism about What?'. Philosophy of Science 58(2), 185-202.

Katzav, J. (2004). 'Dispositions and the principle of least action'. Analysis 64 (283), 206-14.

Ladyman, J. (1998). 'What is structural realism?'. Studies In History and Philosophy of Science Part A 29(3), 409 - 424.

Ladyman, J. and D. Ross (2007). Every thing must go. Oxford University Press.

Landry, E. (2007). 'Shared structure need not be shared set-structure'. Synthese 158(1), $1-17$.

Laudan, L. (1981). 'A confutation of convergent realism'. Philosophy of Science 48, 19-49.

Markosian, N. (2011). Time. In E. Zalta (Ed.), Stanford Encyclopedia of Philosophy. $<$ plato.stanford.edu/entries/time $>$.

Marsden, J. E. and T. S. Ratiu (1995). 'Introduction to mechanics and symmetry'. Physics Today $48(12), 65$.

Muller, F. A. (1997a). 'The equivalence myth of quantum mechanics-Part I'. Studies in History and Philosophy of Science Part B: Studies in History and Philosophy of Modern Physics 28(1), 35-61.

Muller, F. A. (1997b). 'The equivalence myth of quantum mechanics - part II'. Studies in History and Philosophy of Science Part B: Studies in History and Philosophy of Modern Physics 28(2), 219-47.

North, J. (2009). 'The "Structure" of Physics: A Case Study?'. The Journal of Philosophy 106, 57-88.

Pooley, O. (2006). 'Points, particles and structural realism'. In S. French, D. Rickles, and J. Saatsi (Eds.), Structural Foundations of Quantum Gravity, pp. 83-120. Oxford: Oxford University Press.

Psillos, S. (1999). Scientific Realism: How Science Tracks Truth. London: Routledge.

Rickles, D. (2008). The Ashgate companion to contemporary philosophy of physics. Ashgate Publishing.

Ruetsche, L. (2011). Interpreting Quantum Theories. Oxford: Oxford University Press.

Schrödinger, E. (1926). 'Über das Verhältnis der Heisenberg-Born-Jordanschen Quantenmechanik zu der meinem'. Annalen der Physik 384(8), 734-756.

Smart, B. (2012). A Critique of Humean and Anti-Humean Metaphysics of Cause and Law. $\mathrm{Ph}$. D. thesis, Univeristy of Nottingham.

Thébault, K. P. (2012). 'Symplectic reduction and the problem of time in nonrelativistic mechanics'. The British Journal for the Philosophy of Science 63(4), 789-824.

Thebault, K. P. Y. and B. T. Smart (2013). 'On the Metaphysics of Least Action', < philsciarchive.pitt.edu/9642/>.

van Fraassen, B. C. (2006). 'Structure: Its shadow and substance'. The British Journal for the Philosophy of Science 57(2), 275-307.

van Fraassen, B. C. (2008). Scientific Representation: Paradoxes of Perspective. Oxford: Oxford University Press.

Woodhouse, N. (1997). Geometric Quantization. Oxford: Clarendon Press. 
Worrall, J. (1989). 'Structural Realism: The Best of Both Worlds?'. Dialectica 43(1-2), 99-124.

Wüthrich, C. (2010). 'No Presentism in Quantum Gravity'. In V. Petkov (Ed.), Space, Time, and Spacetime: Physical and Philosophical Implications of Minkowski's Unification of Space and Time, pp. 257-78. 